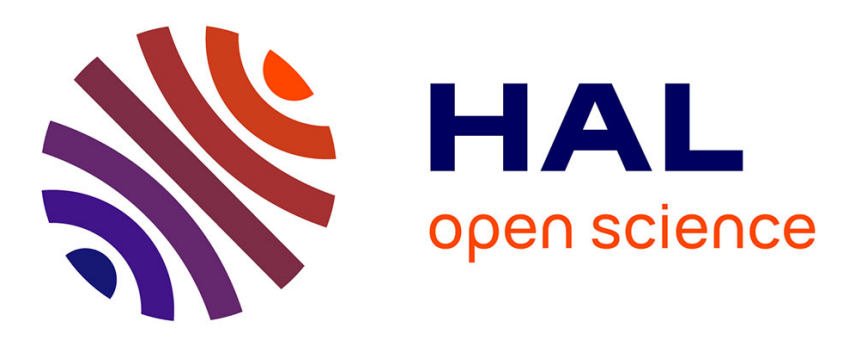

\title{
Coupling filtration and flow during liquid composite molding: Experimental investigation and simulation
}

Delphine Lefevre, Sébastien Comas-Cardona, Christophe Binetruy, Patricia

Krawczak

\section{- To cite this version:}

Delphine Lefevre, Sébastien Comas-Cardona, Christophe Binetruy, Patricia Krawczak. Coupling filtration and flow during liquid composite molding: Experimental investigation and simulation. Composites Science and Technology, 2009, 69 (13), pp.2127-2134. 10.1016/j.compscitech.2009.05.008 . hal-01689204

\section{HAL Id: hal-01689204 https://hal.science/hal-01689204}

Submitted on 21 Jan 2018

HAL is a multi-disciplinary open access archive for the deposit and dissemination of scientific research documents, whether they are published or not. The documents may come from teaching and research institutions in France or abroad, or from public or private research centers.
L'archive ouverte pluridisciplinaire HAL, est destinée au dépôt et à la diffusion de documents scientifiques de niveau recherche, publiés ou non, émanant des établissements d'enseignement et de recherche français ou étrangers, des laboratoires publics ou privés.

\section{(c)(1)}

Distributed under a Creative Commons Attribution| 4.0 International License 


\title{
Coupling filtration and flow during liquid composite molding: Experimental investigation and simulation
}

\author{
D. Lefevre, S. Comas-Cardona, C. Binetruy, P. Krawczak \\ Polymers and Composites Technology and Mechanical Engineering Department, Ecole des Mines de Douai, 941 rue Bourseul, BP 10838,59508 Douai Cedex, France
}

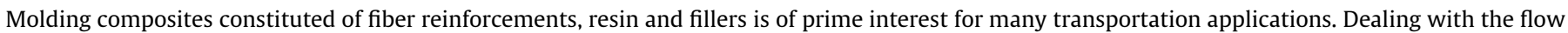

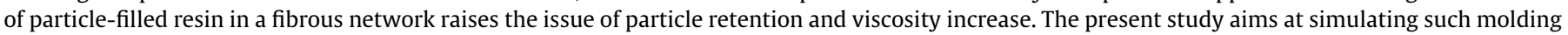

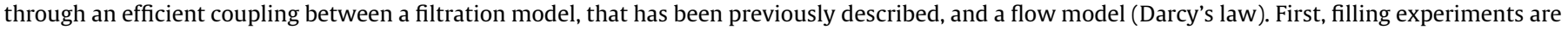

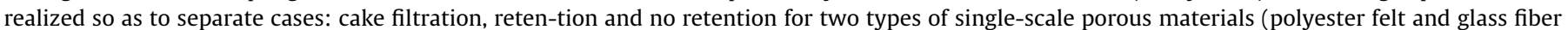

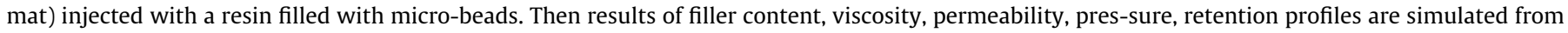

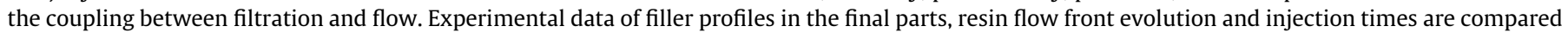
with predic-tions obtained from the simulation.
\end{abstract}

Keywords: Particle-reinforced composites, Modeling, Filtration, Flow

\section{Introduction}

Some large and complex shaped composite parts are produced using Liquid Composite Molding processes (LCM), such as Resin Transfer Molding (RTM) or Liquid Resin Infusion (LRI). In such technologies, a dry fibrous preform is first placed in a mold and the liquid resin is forced through it. Once resin cure is completed, the part is demolded. Automation, low COV emission and reproducibility are some of the advantages of LCM processes.

Manufacturers often blend resins and fillers for specific purposes. Some fillers decrease the overall part cost, others induce upgraded properties. Two classes of particles must be distinguished:

- Nano-scale particles, for which filler content is usually kept low.

- Micron-scale particles (e.g., calcium carbonate) for which filler content may vary from a few percent to $40 \mathrm{vol} \%$ or more.

Dealing with particle-filled resin in LCM processes induces an increase of viscosity and possible filtration of the particles by the fibrous preform. Both phenomena slow the resin flow down and lead towards longer production cycle time or in the worst cases clogging of the fiber reinforcement. Those issues are a major concern especially in mass transportation manufacturing where fire and smoke tolerances of parts are tight. For that type of application, a minimum of $30 \mathrm{vol} \%$ of filler is most of the time required.

For rather high particle content parts, the fibrous reinforcements are typically mats or hybrid materials (a felt or a non-woven

Corresponding author.

E-mail address: comas-cardona@ensm-douai.fr (S. Comas-Cardona). material stitched to a mat) because they exhibit high permeability due to their microstructural openness.

The present paper investigates and simulates the RTM molding of a composite part consisting of a particle-filled resin and a continuous fibrous media. The study will be limited to microscopic fillers and to unidirectional flow. Following a background on particlefilled liquid viscosity evolution and filtration, an experimental investigation of the particle filtration during the injection of composite parts is presented. Then, the modeling of the physical phenomena coupling (i.e., suspension viscosity evolution, flow through porous media and filtration) is given and implemented. Finally a $1 \mathrm{D}$-molding simulation is realized and compared to experimental results.

\section{Background}

\subsection{Viscosity evolution of a particle-filled resin}

Numerous studies have investigated the evolution of suspension (liquid + filler) viscosity with respect to filler content. It has previously been stated that the suspension viscosity increases when:

- the filler content increases [8,4],

- small particles rather than coarse ones are used [19],

- irregular shaped, rough surfaced particles rather than smooth spherical ones are employed [11].

Coussot and Ancey have also observed that a Newtonian suspending liquid displays a shear-thinning behavior with the 


\section{Nomenclature}

$\beta \quad$ entrapped liquid coefficient

$\beta_{0} \quad$ initial value of $\beta$

$\delta \quad$ inter-fiber distance

$\varepsilon \quad$ porosity of the filter

$\varepsilon_{0} \quad$ initial porosity of the filter (clean bed filter)

$\dot{\gamma} \quad$ shear rate

$\phi_{f} \quad$ fiber volume fraction

$\eta \quad$ suspension viscosity

$\eta_{r} \quad$ relative viscosity

$\eta_{0} \quad$ pure resin viscosity

$\Psi \quad$ particle content in the final part

$\sigma \quad$ retention i.e. ratio of the volume of deposited particles to the total volume

$C$ concentration of the moving suspension

$C_{0} \quad$ inlet concentration of the suspension

$d \quad$ particle diameter

$D \quad$ filter grain diameter

$K \quad$ fiber reinforcement with retained particle permeability
$K_{d} \quad$ deposit (retained particles network) permeability

$K_{F} \quad$ initial fiber reinforcement permeability

$k_{0} \quad$ filtration coefficient

$L \quad$ total length of the fibrous medium

m constant

$n \quad$ power-law index

$p \quad$ pressure

$P_{i n j} \quad$ injection pressure

$r \quad$ constant

$s \quad 1 / n$

$t \quad$ time

$T \quad$ filling time

$U \quad$ suspension velocity (Darcy's velocity)

$\bar{v} \quad$ average velocity

$v^{\max } \quad$ maximal velocity

$V \quad$ interstitial velocity of suspension

$x \quad$ 1D coordinate presence of fillers [6]. The effect is even magnified when the filler content is sufficient and allows particles interaction and clustering. Other studies have shown that a blend of $25 \%$ of "fines" with $75 \%$ of "coarse particles" where the diameter ratio of coarse particles to fines is at least seven provides a rather good optimization of the suspension viscosity [9]. It should be noted that optimization lead to satisfactory effects for filler content greater than $40 \mathrm{vol} \%$.

\subsection{Experimental investigations of flow of a particle-filled liquid in a porous media}

The flow of a particle-filled liquid in a fibrous medium is dictated by both the filler size and the fibrous pore size. Experimentally, three different behaviors can be distinguished:

- Cake filtration: The particle size is larger than a critical size that depends on the porous medium characteristics. The particles accumulate onto the medium and form a cake. Thus, particles will not penetrate into the medium and surface filtration occurs.

- No retention: Particles are very small compared to the pore size. The suspension flows easily in the openness of the medium; there is no or very little retention.

- Retention: The suspension flows within the medium, but it progressively captures particles (retention). This phenomenon is called deep filtration. In some cases, the amount of retained particles is such that the network is clogged-up.

Obviously cake filtration does not have industrial interest for composite manufacturing since fillers never enter the fibrous reinforcement. No retention is the ideal case, where all particles can enter the fibrous reinforcement and be well distributed, but that case rarely occurs because a large content of small particles induces very high viscosity leading to very high cycle times or even a difficulty to fully impregnate parts. Retention is the case that is most likely to happen in industrial applications. Because of retention and filtration, filler content and, thus, the subsequent property (e.g., fire resistance) are not homogeneous throughout the composite part, which may become defective. In the most severe conditions when the use of high fiber content fabrics or high filler content is necessary, mold filling becomes difficult or hindered.

According to Sakthivadivel [16], the ratio $D / d$ (where $D$ and $d$ are, respectively, the filter grain and particle diameters) mostly influences the flow of a suspension in a granular porous media.
That study has shown that when $D / d<10$ cake filtration occurs; for $10<D / d<20$ retention takes place and if $D / d>20$ no or limited retention is expected. However, in the case of fibrous media, the definition of $D$ is less obvious since there are no filter grains.

\subsection{Filtration}

Filtration phenomena have been extensively studied in several industrial fields such as water treatment [10], oil well recovery [2], or paper industry [17]. Recent studies have coupled cake formation, particle shapes and fluid flow [13]. Concerning the filtration of particle-filled resin through a fibrous preform during composite processes, some models and investigations have also been published. Filtration models were based on macroscopic approaches coupling kinetics of retention to conservation of mass $[10,7,12]$. Chohra et al. used a geometric and probabilistic model to represent retention of particles in the thickness direction during infusion process [5]. Using particle image velocimetry (PIV), a more recent study observed the preferential retention sites and mechanisms involved during composite molding when particle-filled resin flows in a fibrous media [14]. Lately, experimental studies have focused on filtration of nano-particles in fibrous reinforcements [1]. Hence, both experimental and theoretical studies have been published on these particular matters.

\subsection{Flow of non-Newtonian fluid in a porous media}

As mentioned in a detailed review and previous works, several methods can be used to deal with such flows: capillary bundle approach or drag theory for instance [3] or homogenization [15]. However, those methods are not easy to work with because they require to input parameters such as tortuosity or dynamic specific area and also because the equations are solved on periodic cells which are not well defined for random materials.

\subsection{Motivation}

Even though filtration models exist, no study has coupled them to a flow solver and to resin viscosity evolution when retention occurs during composite materials processing. The retention of particles in the preform will change the porosity and, thus, the permeability of the preform and the resin flow will be affected. Moreover, since the fibrous medium progressively retains particles 
of the suspension, the quantity of particles in the suspension decreases and influences the viscosity. Therefore, the resin flow simulations should be implemented including those variations with respect to time and position. This paper proposes to simulate the injection molding of a composite part consisting of a fiber reinforcement, fillers and resin that includes flow, filtration and material property evolution.

\section{Experimental investigations}

\subsection{Materials}

The suspension is constituted of an unsaturated polyester (UP) resin and spherical micro-beads. The UP resin (Crystic 3027 LV, Scott Bader) has a viscosity of $0.2 \mathrm{~Pa} \mathrm{~s}$ at room temperature $\left(20^{\circ} \mathrm{C}\right)$ and a density of $1.2 \mathrm{~g} / \mathrm{cm}^{3}$. For fillers, spherical glass or ceramic micro-beads (3M, Sovitec, Potters) were preferred to irregular shaped particles so as to only modify size and not overall shape. Their physical properties are presented in Table 1 . When micro-beads and resin are blended, $1.5 \%$ of additive (BYK-W 980, BYK-Chemie) is used to limit filler sedimentation and aggregation.

A synthetic PET fiber felt and an E-glass fiber mat have been used. Such materials have been chosen because they are representative of the materials used when dealing with high filler content applications such as fire resistance in mass transportation parts. The fiber reinforcement properties are given in Table 2 . The PET fiber felt has also been chosen for two reasons: it is a single-scale porous media and also it is completely eliminated after sample burning, so filler content profiles can be easily determined in the final parts. In order to achieve fiber volume fractions ranging between $10 \%$ and $25 \%$, the number of plies layed-up in the mold cavity varied from 1 to 4 for the felt and from 3 to 5 for the mat.

\subsection{Viscosity measurements}

Viscosity measurements have been carried out with a Couette device mounted on a rotational rheometer ARES (Rheometric Scientific). Relative viscosities $\eta_{r}$ (Eq. (1)) have been measured for filler content ranging between $0 \mathrm{vol} \%$ and $50 \mathrm{vol} \%$ and shear rates between 1 and $100 \mathrm{~s}^{-1}$. The corresponding results are depicted in Fig. 1. As expected, the viscosity increases with filler content, and displays a shear-thinning behavior.

$\eta_{r}=\frac{\eta}{\eta_{0}}$

where $\eta$ is the viscosity of the particle-filled suspension and $\eta_{\mathrm{o}}$ the one of pure resin. Pipe flow experiments have also been performed and show no migration of fillers.

\subsection{Experimental setup and procedure}

The experimental setup is composed of a rigid tooling (steel half mold and thick PMMA top plate). The mold cavity is $90 \mathrm{~mm}$ wide,

Table 1

Physical properties of micro-beads.

\begin{tabular}{lllll}
\hline Volume average diameter $(\mu \mathrm{m})$ & 3 & 12 & 30 & 48 \\
\hline Material & Ceramic & Glass & Glass & Glass \\
Density $\left(\mathrm{g} / \mathrm{cm}^{3}\right)$ & 2.5 & 2.46 & 2.54 & 2.46 \\
\hline
\end{tabular}

Table 2

Physical properties of fiber reinforcements.

\begin{tabular}{lll}
\hline Fiber reinforcement & E-glass fiber mat & PET fiber felt \\
\hline Fiber density $\left(\mathrm{g} / \mathrm{cm}^{3}\right)$ & 2.54 & 1.39 \\
Reinforcement areal weight $\left(\mathrm{g} / \mathrm{m}^{2}\right)$ & 450 & $300-400$ \\
\hline
\end{tabular}

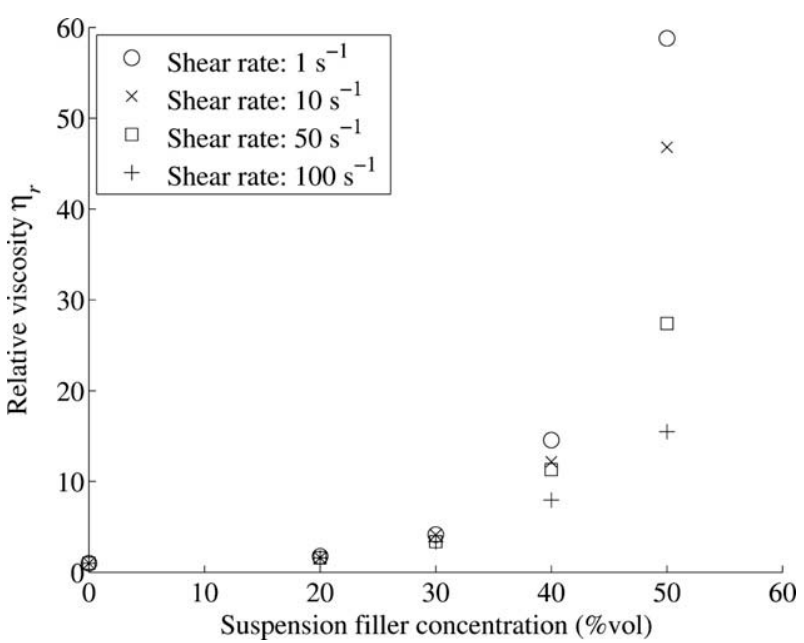

Fig. 1. Relative viscosity of suspensions (resin $+12 \mu$ m-particles) with respect to shear rate and particle concentration.

$4 \mathrm{~mm}$ thick and $400 \mathrm{~mm}$ long. The mold is fed at constant pressure (0.2 MPa) using a pressure bucket, equipped with a motorized mixer so as to maintain a homogeneous blend of the suspension (Fig. 2).

Two types of experiments were run. First, the experiments consist in impregnating a preform of length $L$ with the suspension (resin and particles). Several experiments have been run while modifying fiber volume fraction, particle diameter and particle content. Because of the large amount of experiments, instead of repeating several times the same experiment, the choice of realizing many experiments in the $d, C_{0}$, $\phi_{f}$ domain has been made. Both fiber mat and felt have been tested. The flow is monitored through the PMMA mold top plate that allows observations. The corresponding results are given in Section 3.4.

The second type of experiments deals with injecting composite parts with the same setup, however, the fibrous reinforcement is the felt $\left(\phi_{f}=17 \%\right)$ and the polyester resin is filled with microbeads. During the injection, the flow front positions are recorded with respect to time and the injection time is measured. Once the resin has cured, the part is demolded. Samples are cut at various locations and are submitted to a burn-off test procedure so as to evaluate the filler content profile in the final part. Readers may refer to Lefevre et al. [12] for details about that procedure. The results obtained are compared to simulation in Section 6 .

For both types of experiments the top lid of the mold deflection has been checked and remained below $100 \mu \mathrm{m}$ during the mold filling.

\subsection{Experimental results}

The modification of fiber volume fraction, filler content and particle diameter influenced the flow of the suspension in the porous media. As expected, three cases occurred:

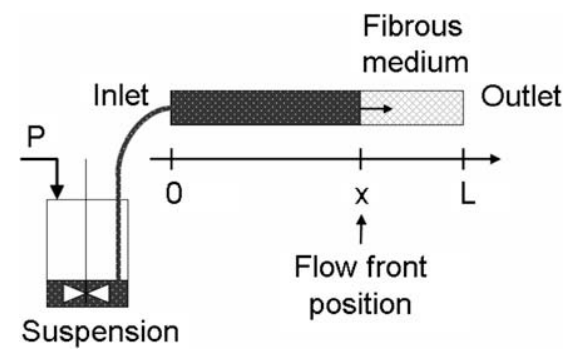

Fig. 2. Experimental setup. 
- Cake filtration: The particles accumulate ahead of the fiber reinforcement as soon as the injection starts, only resin flows within the reinforcement.

- Progressive clogging (retention): The suspension flows within the fiber reinforcement for some time, particles accumulate into the porous medium and then cake filtration starts.

- Flow (no retention): The suspension flows within the fiber reinforcement.

The results have been depicted in Fig. 3 where each experiment is reported with a specific marker (circle, triangle or cross). It is common to relate filtration phenomena to particle diameter, thus Fig. 3 depicts the results in that sense ( $x$-axis is the particle diameter $d$ ). For the two reinforcements, when fiber volume fraction and particle size increase, the flow is most of the time hindered. The large particles cannot flow easily in the space left between the fibers. For small particles and low volume fraction, the suspension flows easily. However, some experiments (e.g., $50 \mu \mathrm{m}$-diameter particles and 25 vol\% fiber volume fraction in Fig. $3 \mathrm{~b}$ ), show either flow or immediate clogging. The difference during such experiments came from the particle content. Considering only fiber volume fraction and particle size when plotting experimental results lacks interest since clogging may also appear with suspension clustering when the particle content is high.
Therefore, the experimental results have been plotted using modified axes in Fig. 4. Because both particle size $d$ and suspension concentration $C_{0}$ represent the volume of particle flowing through the fibrous medium, it would be more relevant to plot the experimental data as a function of the variable $d C_{0}$, as shown in Fig. 4. With this axis, the experimental data are spread out more evenly in the $\left(d C_{0}, \phi_{f}\right)$ domain. For low $\phi_{f}, d$ or $C_{0}$ the suspension flows easily. Then, when particle size or suspension concentration or fiber volume fraction increases, retention increases and the fibrous medium gradually clogs up. For high values of $\phi_{f}, d$ or $C_{0}$ clogging (cake filtration) occurs instantaneously.

\section{Modeling}

\subsection{Coupling}

When dealing with injection of particle-filled resin within fiber reinforcements, several phenomena take place and are coupled. For instance, the suspension velocity depends on fibrous reinforcement porosity and permeability and of course on the viscosity of the suspension. Nonetheless, viscosity depends on the filler content, the latter depending on filtration, which also depends on flow velocity. Fig. 5 shows in detail the coupling between flow and filtration, and how parameters and variables are coupled and

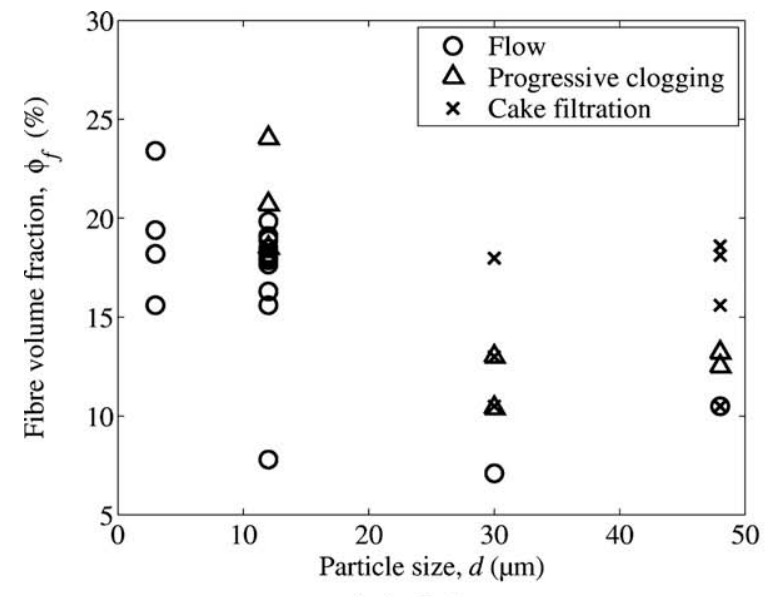

(a) felt

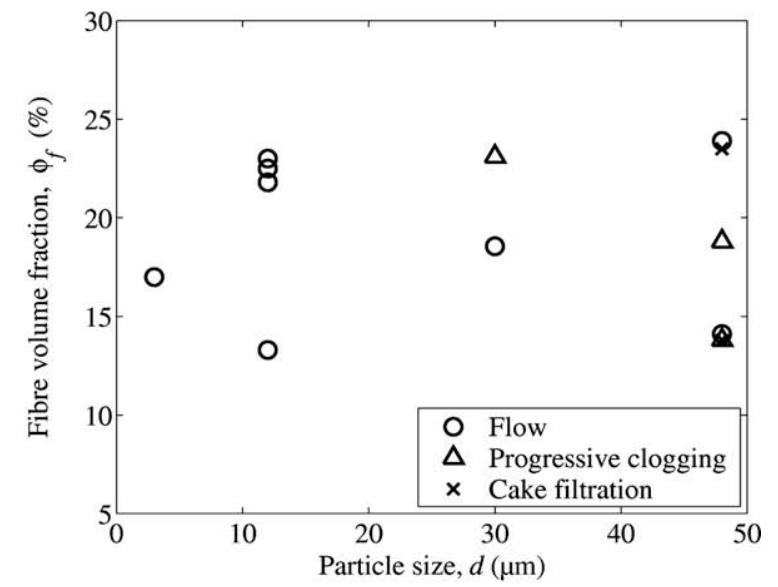

(b) mat

Fig. 3. Visual observations during molding plotted in the $\left(d, \phi_{f}\right)$ domain.

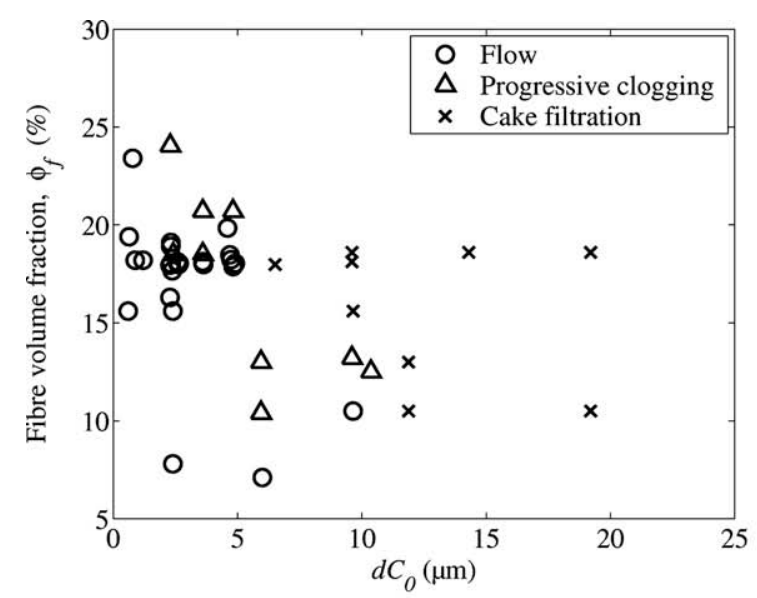

(a) felt

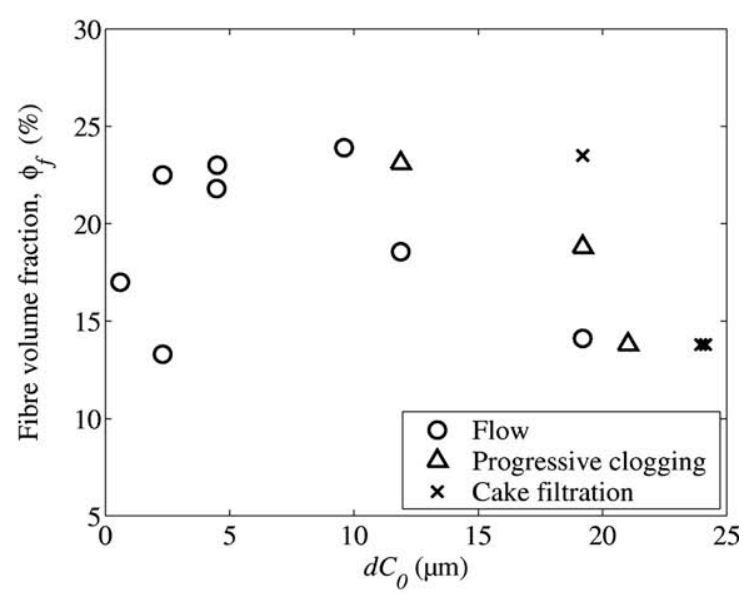

(b) mat

Fig. 4. Visual observations during molding plotted in the $\left(d C_{0}, \phi_{f}\right)$ domain. 


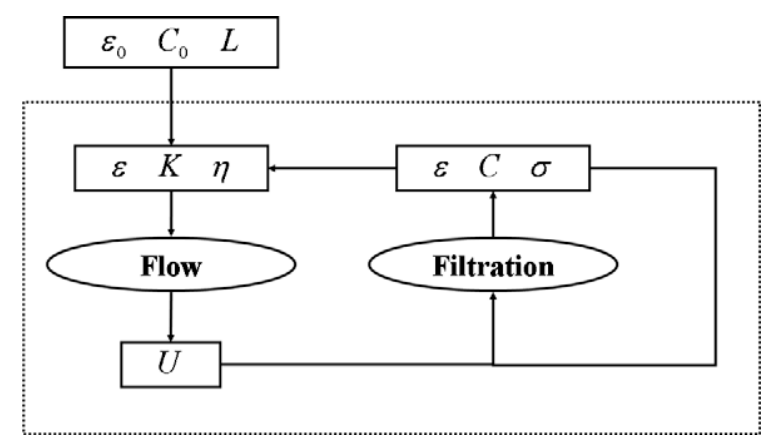

Fig. 5. Flow and filtration coupling during Liquid Composite Molding processes.

affected. The suspension flows in the reinforcement with a velocity $U$. In some cases, filtration and retention $\sigma$ may occur and lead to local variations of porosity $\varepsilon$ and particle suspension concentration C. Such modifications will also lead to changes in viscosity $\eta$ and permeability $K$, which will affect flow velocity. In the following, the details of the modeling (flow, filtration and properties update) are given.

\subsection{Flow sub-model}

Darcy's law (Eq. (2)) has been widely used as the momentum equation to model flow through porous media. The suspension interstitial velocity is $V(t, x)$, varies with the porosity of the filter and is linked to the Darcy's velocity $U$. Even though the suspension has a non-Newtonian behavior (Fig. 1), since the filtration model is macroscopic, the viscosity $\eta$ will be considered constant within an element of the discretized domain. Details of the viscosity estimate are given in Section 4.4.1.

$U=V \varepsilon=-\frac{K}{\eta} \nabla p$

where $\nabla p$ is the pressure gradient.

\subsection{Filtration sub-model}

The understanding of the occurrence of filtration in Liquid Composite Molding processes will be realized using a macroscopic model for filtration. The main equations are given in this section; the readers may refer to Lefevre et al. [12] for details in derivations and assumptions of the model.

All molding experiments have been unidirectional; therefore the problem is reduced to a 1D domain. As the suspension flows, particles are retained in the fibrous medium. This phenomenon is called the retention and is expressed by $\sigma$, which is the volume fraction of retained particles in the filter volume. The porosity of the filter $\varepsilon$ available to the suspension decreases with time as it is clogged by particles, i.e.:

$\varepsilon=\varepsilon_{0}-\beta \sigma$

where $\beta$ represents the presence of entrapped liquid between the retained particles or between the retained particles and the fibrous medium. That entrapped liquid will neither participate in the flow of the suspension nor in the dilution. For dilute suspension and limited retention, the entrapped liquid can be neglected in the equations, but in this study, the concentrations and retention can reach high levels and requires taking into consideration such volume. Furthermore, it is also assumed that $\beta$ is not constant during the injection and that it directly depends on the retention $\sigma$ :

$\beta=\beta_{0}-r \sigma$ where $\beta_{0}$ is the initial value of $\beta$ and $r$ is a constant. More details on the effect on the $\beta$ coefficient on the retention profiles are given in [12].

Assuming no diffusion and no physico-chemical interactions, the mass balance equation can be written as:

$\frac{\partial(\sigma+\varepsilon C)}{\partial t}+U \frac{\partial C}{\partial x}=0$

Substituting Eqs. (3) and (4) into Eq. (5) leads to:

$\varepsilon \frac{\partial C}{\partial t}+U \frac{\partial C}{\partial x}=\left(\beta_{0} C-2 r C \sigma-1\right) \frac{\partial \sigma}{\partial t}$

A constitutive equation for particle deposition kinetics is chosen as follows:

$\frac{\partial \sigma}{\partial t}=k_{0} U C$

Eq. (7) assumes that the deposition rate is proportional to the particles present and conveyed in the suspension, i.e. UC. The proportionality coefficient $k_{0}$ is called the filtration coefficient. Therefore, the filtration sub-model consists of two governing equations (Eqs. (3) and (6) and two constitutive equations (Eqs. (4) and (7)) that are used to solve for the unknowns of the problem: $C, \sigma, \beta$ and $\varepsilon$.

\subsection{Properties update}

Since material properties (suspension viscosity and permeability) evolve with time and position, it is necessary to evaluate them at each time step.

\subsubsection{Viscosity}

The suspension viscosity depends on both filler concentration and shear rate. Therefore, a double interpolation is necessary and performed based on those two parameters. The filler concentration profile is known at each time step from the filtration sub-model. Regarding shear rate, which is unknown, it will be estimated from the Darcy's velocity $U$ as a first approach. The local average shear rate in the porous media is approximated from the velocity profile of a power-law fluid in a tube whose dimensions are representative of the porous media. The flow of a power-law fluid in a pipe shows that the average velocity $\bar{v}$ is linked to the maximum velocity $v^{\text {max }}$ by:

$v^{\max }=\bar{v} \frac{s+3}{s+1}$

where $s=1 / n$ and Table 3 lists the values of the power-law index $n$. The index $n$ has been obtained from a fit of the viscosity data displayed in (Fig. 1) to the power-law relationship:

$\eta=m \dot{\gamma}^{n-1}$

The average velocity $\bar{v}$ can be approximated to the interstitial velocity in the fibrous medium:

$\bar{v} \cong V=\frac{U}{\varepsilon}$

and similarly for the average shear rate:

$\dot{\gamma} \cong \frac{v^{\max }}{\delta / 2}$

Table 3

Values of the power-law index $n$ of the suspension as a function of $C_{0}$.

\begin{tabular}{lll}
\hline$C_{0}($ vol\%) & $n$ & $\frac{s+3}{s+1}$ with $s=1 / n$ \\
\hline 20 & 0.992 & 1.99 \\
30 & 0.926 & 1.96 \\
40 & 0.866 & 1.93 \\
50 & 0.708 & 1.83 \\
\hline
\end{tabular}


where $\delta$ is the average distance between fibers. The average distance between fibers in a mat or felt can be estimated by [18]:

$\delta=\sqrt{K}$

where $K$ is the permeability of the porous media (fibers and retained fillers) which evolves with respect to time and position. Substituting Eqs. (8), (10), and (12) into (11), the estimated average shear rate of the flow in the porous media becomes:

$\dot{\gamma} \cong \frac{\bar{v}\left(\frac{s+3}{s+1}\right)}{\delta / 2} \cong \frac{4 U}{\delta \varepsilon}=\frac{4 U}{\sqrt{K} \varepsilon}$

\subsubsection{Permeability}

Regarding permeability, since the fibrous preform retains fillers, the new filter system (fibrous preform + retained particles) has a permeability that has to be evaluated. Assuming perfect saturation of the new filter system, the equivalent permeability $K$ is the average permeability of the two systems in parallel. The permeability of this evolving filter system is given by:

$\frac{1}{K}=\frac{1}{K_{F}}+\frac{1}{K_{d}}$

The permeability of the fiber felt $\left(\phi_{f}=17 \%\right)$ is $K_{F}=4.92 \times 10^{-10} \mathrm{~m}^{2}$ and has been measured on an injection permeability bench. The deposit permeability $K_{d}$ can be estimated using the Kozeny-Carman relationship:

$K_{d}=\frac{d^{2}(1-\sigma)^{3}}{36 h_{k} \sigma^{2}}$

where $36 h_{k}=150$ for granular media.

\section{Simulation}

All equations described previously will be implemented to simulate 1D filling of the composite parts made experimentally.

\subsection{Flow chart}

The general flow chart of the simulation is given in Fig. 6. Input parameters are constant and depend on material, geometrical and technological considerations:

- Length of the mold $L$, injection pressure $P_{i n j}$.

- Fiber volume fraction $\phi_{f}$ (or initial porosity $\varepsilon_{0}$ ), average distance between fibers $\delta$, fiber reinforcement permeability $K_{F}$.

- Filtration coefficient $k_{0}$, initial liquid entrapment coefficient $\beta_{0}$, parameter $r$.

- Initial filler concentration $C_{0}$.

The values of the input parameters are given in Table 4 .

The domain is unidirectional and has 101 nodes (100 1D elements). The time step $d t$ is not constant during the simulation and is calculated so as to exactly fill the next 1D element. Because the experimental injections are realized at constant pressure, the flow velocity decreases and $d t$ increases with the mold filling (i.e. with time).

The main calculation loop (Fig. 6) contains three main steps which are successively solved at each time step:

- Flow sub-model: A finite difference solver for Darcy's law (Eq. (2)) (including spatially non-constant viscosity and permeability) has been developed using Matlab (The MathWorks). The

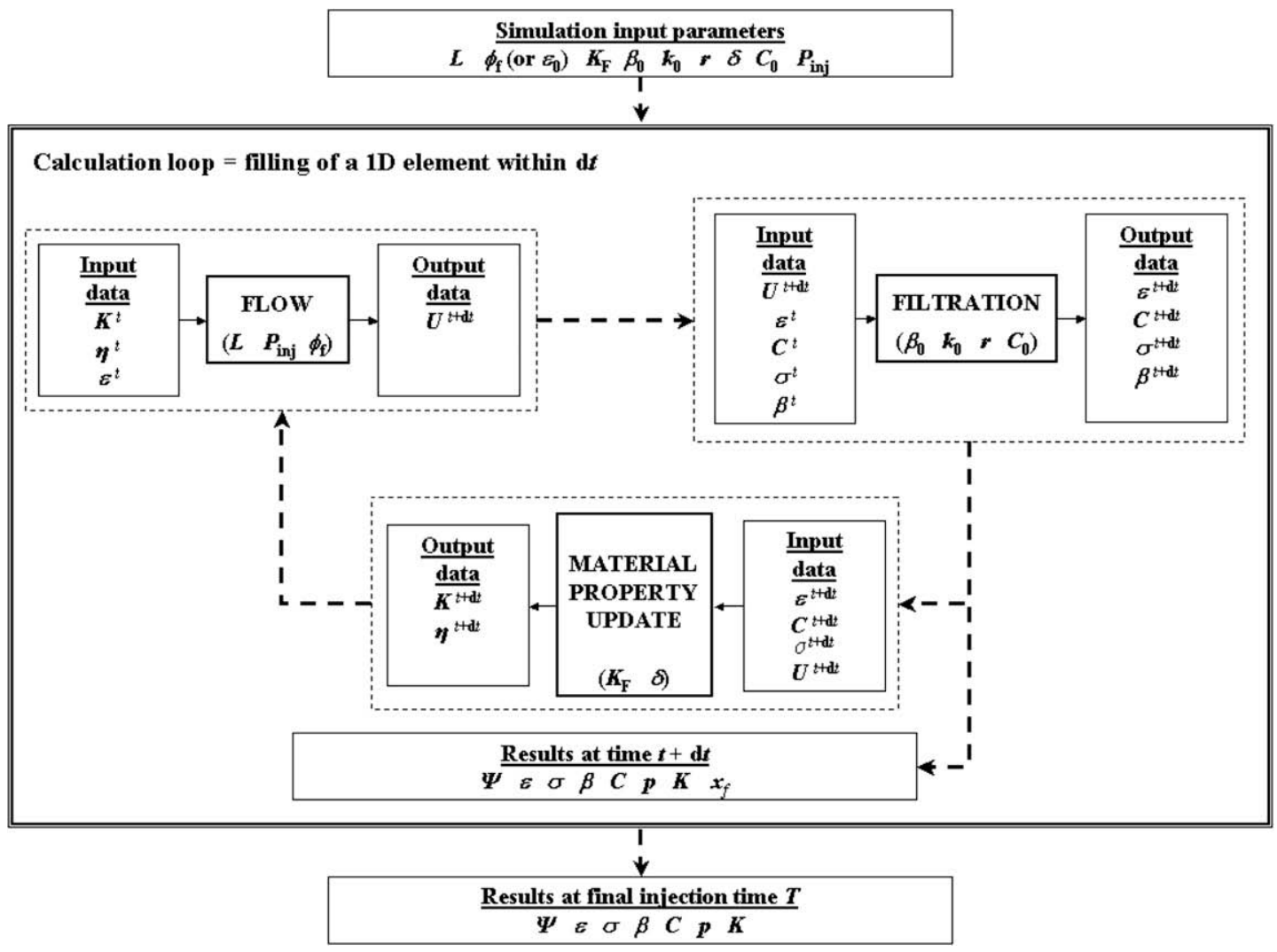

Fig. 6. Flow chart of the simulation of a particle-filled resin injection through a fibrous preform. 
Table 4

Parameters used in the simulations.

\begin{tabular}{llll}
\hline Parameter & & Value & \\
\hline$C_{0}$ & $21.5 \%$ & $30.3 \%$ & $40.5 \%$ \\
$L$ & & $0.2 \mathrm{~m}$ & \\
$\phi_{f}$ & 0.187 & \\
$\varepsilon_{0}$ & & 0.813 & \\
$K_{F}$ & $4.92 \times 10^{-10} \mathrm{~m}^{2}$ & \\
$\beta_{0}$ & 6.5 & 5.15 & \\
$k_{0}$ & & $0.0157 \mathrm{~cm}^{-1}$ & \\
$r$ & & 30 & \\
$\delta$ & & $\sqrt{K}(\mathrm{Eq} .(14))$ & \\
$d$ & & $12 \mu \mathrm{m}$ & \\
$P_{i n j}$ & $0.2 \mathrm{MPa}$ & \\
\hline
\end{tabular}

main inputs are: permeability $K_{F}$, viscosity $\eta$, porosity $\varepsilon$, length $L$ of the mold and injection pressure $P_{i n j}$. The output of that solver is the suspension velocity $U$.

- Filtration sub-model: A finite element method is used to solve for Eqs. (3), (4), (6), and (7). A commercial code (COMSOL Multiphysics 3.3 , COMSOL $A B$ ) is used. The input parameters are the suspension velocity $U$, the porosity $\varepsilon$, the retention $\sigma$ and the filler concentration $C$ profiles. The filtration coefficient $k_{\mathrm{o}}$, fluid entrapment coefficient $\beta$ and constitutive equation parameter $r$ are the ones already obtained in a previous study for identical materials (see [12] for details). The outputs are the porosity $\varepsilon$, the retention $\sigma$ and the suspension $C$ concentration profiles. The code has been validated in the case when no particles are present and compared to the 1D Darcy's analytical solution at constant injection pressure. Also both influence of the mesh refinement on the solution and the convergence of the solution have been checked.

- Material properties update: permeability K (Eqs. (14) and (15)) and viscosity $\eta$ (Eqs. (9) and (13)) are updated with time and position from the suspension velocity $U$, porosity $\varepsilon$, retention $\sigma$ and suspension concentration $C$ profiles.
The main loop is implemented using Matlab, the filtration model is called as a slave routine.

\subsection{Initial and boundary conditions}

The boundary condition for pressure in the flow sub-model is $p(x=0, t>0)=P_{i n j}$. Regarding initial conditions, $t=0, p(x, t=0)=0$. For the filtration sub-model, there are initially no particles in the domain and thus, for any location within the media, the particle concentration is $C(x, t=0)=0$ and the retention is $\sigma(x, t=0)=0$. The initial porosity is the one of the clean fiber bed i.e. $\varepsilon(x, t=0)=\varepsilon_{0}$. The boundary conditions are $C(x=0, t)=C_{0}$ at the inlet and free convective flux at the outlet $(x=L)$. However, because of the concentration singularity at $x=0$ and $t=0$, the concentration will be gradually raised from 0 towards $C_{0}$, within the first $10^{-2} \mathrm{~s}$ of the injection, using a Heaviside function.

\section{Results and discussion}

The results from the simulations give various profiles with respect to position and time: permeability, viscosity, porosity, filler concentration, retention as shown in Fig. 7 (with the $C_{0}=30.3$ vol\% injection case). The retention decreases along the part length and induces a variation of permeability as dictated by Eq. (14). Meanwhile, the porosity (space left for the suspension to flow) is lower at the inlet than at the outlet, because of retention. The entrapped liquid coefficient $\beta$ is also lower at the inlet because more and more particles are able to agglomerate in the retained clusters. The suspension concentration profile directly induces the viscosity trend. The pressure profile within the part is no longer linear. It shows a curvature that reflects the combination of permeability and viscosity variations along the part. The final filler profile in the part agrees well with the experimental one. The filler concentration profile is $U$-shaped due to the fact that when particles are retained they also entrapped liquid that neither contributed to
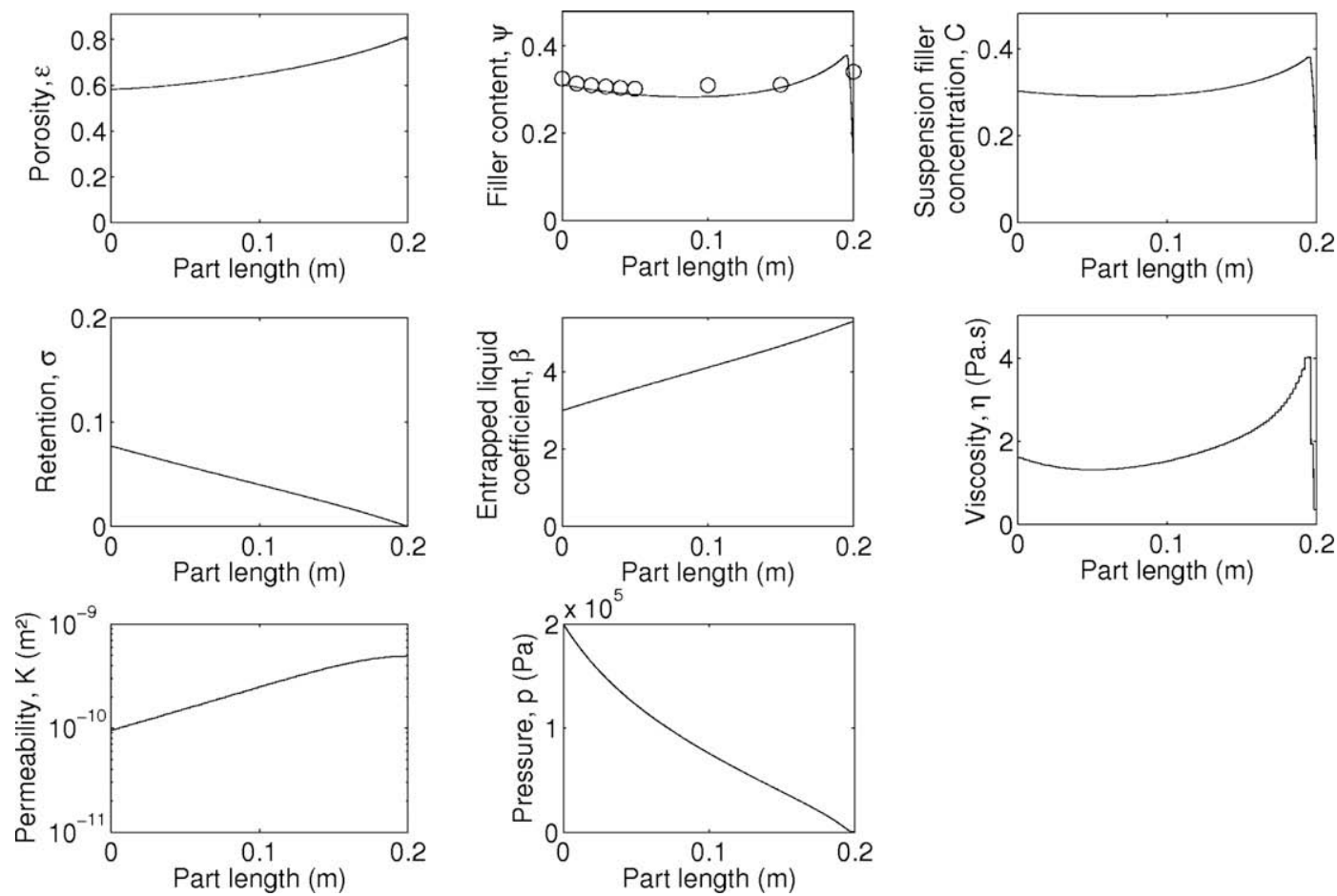

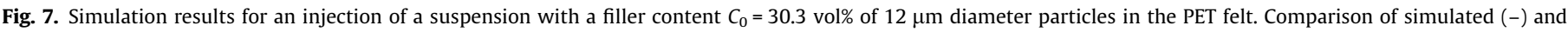
experimental values $(O)$ of filler content profile $\Psi$ in the final part. 


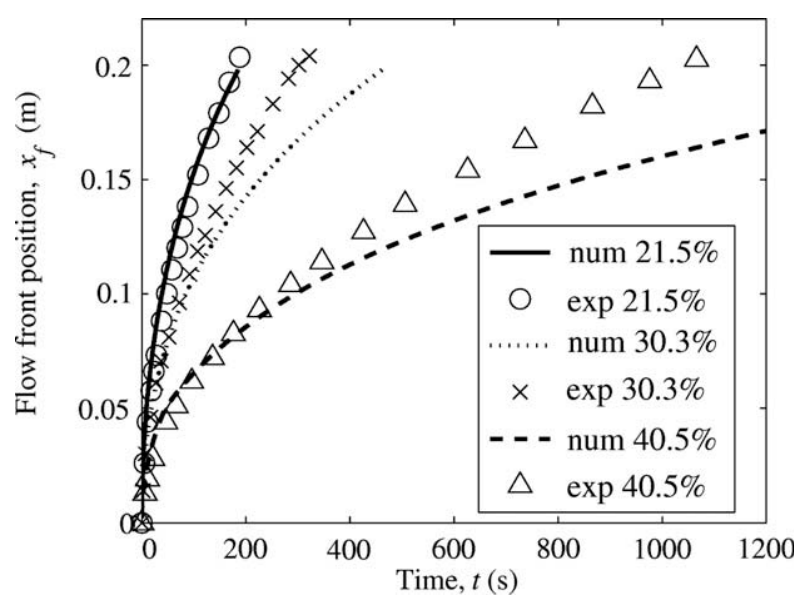

Fig. 8. Comparison of the experimental and simulated results for resin flow front advancement prediction in the case of $C_{0}=21.5,30.3$ and 40.5 vol\%.

Table 5

Predicted and experimental times.

\begin{tabular}{llll}
\hline Filler concentration (vol\%) & 21.5 & 30.3 & 40.5 \\
Predicted injection time $(s)$ & 190 & 474 & 1831 \\
Experimental injection time $(s)$ & 190 & 320 & 1065 \\
\hline
\end{tabular}

the flow nor to the dilution. On micrographs, some locations show resin-rich regions which are much larger that the particle diameter. Therefore, the fluid entrapment induces a filler concentration increase at the front. That effect has been seen for many experiments. Readers may refer to previous published results for more details on that matter [12].

The simulation has also been performed for the two other concentrations $\left(C_{0}=21.5 \mathrm{vol} \%\right.$ and $C_{0}=40.5 \mathrm{vol} \%$ ). The results show very similar trends and profiles, and the final filler contents in the part agree well with the experimental ones.

The flow results from a competition between a low permeability and viscosity at the mold inlet and a high permeability and viscosity at the outlet. The simulation code is also able to predict the flow front advancement for the three filler concentrations as depicted in Fig. 8. The latter shows a rather good agreement for 20 vol\% of filler and starts to deviate for larger filler contents. The predicted injection times are compared to the experimental ones in Table 5 and show a good agreement in terms of order of magnitude.

Deviation between experimental and predicted results may arise from the different assumptions and estimations that have been chosen. As a matter of fact, a small error on permeability or viscosity estimates directly impacts the filling times. Since this work was a first attempt to couple flow and filtration during LCM processes, the assumptions and estimates that have been chosen were rather straightforward. Looking at the results obtained, some of the estimates should be modified and especially the permeability and viscosity ones that directly govern and impact the flow response. Also, it has been chosen to couple the (macroscopic) Darcy's equation with a power law model for viscosity. However, other methods have been studied or are still under investigation so as to handle non-Newtonian flows in porous medium $[3,15]$. Because the choice in this study has been made to build a macroscopic model, the next stage of the study should involve a microscopic modeling coupling power-law flow in a porous medium with a local filtration model.

\section{Conclusions}

The filtration model previously proposed [12] has been coupled to a flow solver to simulate the molding of a resin-filled composite part. This coupling allows to quite precisely predict such molding. Process optimization when dealing with particle-filled resins could be undertaken. Also the code allows the user to distinguish the amounts of moving from retained particles with respect to time and position. Such distinction is interesting to predict because they cannot be easily measured experimentally. The code should also predict when clogging occurs and at what location. Clogging detection is achieved looking at porosity evolution with respect to time: when the porosity is null, there is no more available volume for the suspension to flow. That feature should be validated with several experiments comparing maximal lengths that can be injected for a resin/filler/reinforcement system for instance.

\section{References}

[1] Akinyede O, Sankar J, Mohan R, Kelkar A. Investigation of particulate filtration of alumina nano-particles in VARTM processing of hybrid composites. In: Proceedings of the ASME international mechanical engineering congress and exposition, November 11-15, 2007, Seattle, WA, vol. 13; 2008. p. 157-62.

[2] Alvarez AC, Bedrikovetsky PG, Hime G, Marchesin AO, Marchesin D, Rodrigues JR. A fast inverse solver for the filtration function for flow of water with particles in porous media. Inverse Prob 2006;22:69-88.

[3] Chhabra RP, Comiti J, Machac I. Flow of non-Newtonian fluids in fixed and fluidized beds. Chem Eng Sci 2001;56:1-27.

[4] Cheng N-S, Law AW-K. Exponential formula for computing effective viscosity. Powder Technol 2003;129:156-60.

[5] Chohra M, Advani SG, Gokce A, Yarlagadda S. Modeling of filtration through multiple layers of dual scale fibrous porous media. Polym Comp 2006;27:570-81.

[6] Coussot P, Ancey C. Rhéophysique des pâtes et des suspensions. EDP Sciences; 1999. 264p.

[7] Erdal M, Güçeri S, Danforth SC. Impregnation molding of particle-filled preceramic polymers: process modeling. J Am Ceram Soc 1999;82(8):2017-28.

[8] Greenzweig, JE, Pickering, TL. Flow properties of calcium carbonate filled polyester resins. In: SPI 31st annual technical conference proceedings; 1976. p. 1-8 [section 8-D].

[9] Greenwood R, Luckham PF, Gregory T. Minimising the viscosity of concentrated dispersions by using bimodal particle size distributions. Colloids Surf - A 1998;144:138-47.

[10] Herzig JP, Leclerc DM, Le Goff P. Flow of suspensions through porous media. Application to deep filtration. Ind Eng Chem 1970;62(5):8-35.

[11] Kitano T, Kataoka T, Shirota T. An empirical equation of the relative viscosity of polymer melts filled with various inorganic fillers. Rheol Acta 1981;20:207-9.

[12] Lefevre D, Comas-Cardona S, Binetruy C, Krawczak P. Modelling the flow of particle-filled resin through a fibrous preform in liquid composite molding technologies. Composites: Part A 2007;38:2154-63.

[13] Nazarboland MA, Chen X, Hearle JWS, Lydon R, Moss M. Effect of different particle shapes on the modelling of woven fabric filtration. J Inform Comput Sci 2007;2(2):111-8.

[14] Nordlund M, Fernberg SP, Lundström TS. Particle deposition mechanisms during processing of advanced composite materials. Composites: Part A 2007;38(10):2182-93

[15] Orgeas L, Idris Z, Geindreau C, Bloch J-F, Auriault J-L. Modelling the flow of power-law fluids through anisotropic porous media at low-pore Reynolds number. Chem Eng Sci 2006;61:4490-502.

[16] Sakthivadivel, R. Clogging of a granular porous medium by sediment. University of California, Berkeley, Report HEL 15-7; 1969.

[17] Vengimalla R, Chase GG, Ramarao BV. Modeling of filler retention in compressible fibrous media. Separat Purif Technol 1999;15:153-61.

[18] Ward JC. Turbulent flow in porous media. Proc Amer Soc Civil Eng 1964;90(HY5)

[19] Woychesin EA, Sobolev I. Effect of particle size on the performance of alumina hydrate in glass-reinforced polyesters. In: SPI 30th anniversary technical conference proceedings; 1975 . p. 1-8 [section 4-B]. 